\title{
Kurt Hiibner
}

\section{Zur Politischen Ökonomie des doppelten Deutschland}

\section{Deutsch-deutsche Simulationen}

Politische Ereignisse und Handlungen stimmen nur ausnahmsweise mit einer ökonomischen Logik von Entwicklung überein. Dies kann auch nicht anders sein: Die gesellschaftlichen Subsysteme Politik und Ökonomie sind durch jeweils eigene Prinzipien, Regeln, Normen, Codes und Logiken gekennzeichnet, die sich komplementär verhalten können, aber keineswegs müssen. In der Regel bestehen zwischen beiden Subsystemen Konflikte, Spannungen und Widersprïche, die von Zeit zu Zeit offen zum Ausbruch kommen können. Diese analytische Grundeinsicht wurde durch die spektakulären gesellschaftlichen Umbrüche der Internationalen Politischen Ökonomie der späten achtziger Jahre wieder einmal bestätigt. Ausgerechnet in einer weltökonomischen wie weltpolitischen Konstellation, in der sich die Krise der »Pax Americana in eine offene Hegemoniekrise der USA übersetzt hatte und sich oligopolistische Strukturen einer regionalisierten »westlichen« Weltwirtschaft herauszukristallieren begannen, kumulierten sich die Instabilitäten der Länder des realsozialistischen Blocks zu einem von nur wenigen Beobachtern ernsthaft erwarteten - systemischen Zusammenbruch. Dem Kalten Krieg und den darauf bezogenen westlichen Entwicklungsmodellen waren wesentliche Triebkräfte, der Nachkriegsordnung der Internationalen Politischen Ökonomie sicher geglaubte Fundamente entzogen. Mit dem Zusammenbruch dieser Gesellschaften und ihren Übergangsversuchen zu kapitalistischen Markt- und Geldwirtschaften werden die Konkurrenzverhältnisse der Weltwirtschaft im Zuge einer Neustrukturierung der internationalen Arbeitsteilung neu gestaltet.

Der systemische breakdown der ost- und mitteleuropäischen Gesellschaften erwies sich für die OECD-Gesellschaften als tiefer politischer und ideologischer Orientierungsverlust, der - verschärfend - von einer zyklischen wie strukturellen ökonomischen Krise überlagert wurde. In einer ungünstigeren Konstellation, so ließe sich aus einer rein ökonomischen Perspektive formulieren, hätten der Zusammenbruch der bipolaren Nachkriegsordnung im allgemeinen und die Selbstauflösung der Deutschen De- 
mokratischen Republik als einem Pfeiler dieser Ordnung im besonderen sich nicht ereignen können, fehlen doch den westlichen Markt- und Geldgesellschaften aufgrund drastischer interner Strukturprobleme jetzt nicht nur die finanziellen Ressourcen zur Abstützung der eingeleiteten Transformationsprozesse in Ost- und Mitteleuropa, sondern darüberhinaus auch die politische Strategie, um mit der gewandelten globalen Konstellation produktiv umgehen zu können. Angesichts der tiefgreifenden weltwirtschaftlichen Umbrüche unter Bedingungen verschärfter globaler Konkurrenz drängt sich der Eindruck auf, daß den entwickelten kapitalistischen Ländern der Rock näher als die Hose sitzt. Exkludente Strategien zur Sicherung bzw. zum Ausbau ihrer globalen Konkurrenzpositionen dominieren gegenüber inkludenten Strategien einer Neustrukturierung der globalen Ökonomie, in der sowohl die ost- und mitteleuropäischen Transformationsökonomien als auch die sog. Drittweltökonomien ihren Platz finden könnten (vgl. dazu Cardoso 1993).

Die politischen und ökonomischen Akteure der OECD-Gesellschaften konnten sich nur kurzzeitig über den »systemischen Sieg « des westlichen Kapitalismus freuen. Seit einiger Zeit werden die bislang nur zaghaft erhobenen Stimmen lauter, daß die Ereignisse in Ost- und Mitteleuropa bereits heute negative Konsequenzen für die OECD-Gesellschaften und das globale System der kapitalistischen Markt- und Geldwirtschaft zeitigen, und vor allem noch zeitigen werden. Dies gilt ebenso für die Vertiefungspolitik der Europäischen Union im Zuge der Maastricht-Verträge, die sich starken Erweiterungstendenzen vor allem der geographisch benachbarten Reformstaaten wie Polen, Ungarn und Tschechiens gegenübergestellt sieht, wie für die Standortstrategien der OECD-Staaten, die sich mit den Billiglohnökonomien der Transformationsgesellschaften neuen Herausforderungen gegenübersehen, oder etwa auch für die Umstrukturierung der Arbeitsmärkte im Zuge ost- westlicher Migrationsprozesse. Der deutsch-deutsche Einheitsprozeß simuliert gleichsam - bei allen wichtigen Differenzen im Kleinen wie im Großen - die ost-westliche Interdependenz simultaner Umbruchprozesse, und vermag somit ein Schlaglicht auf die Probleme werfen, die sich aus der weltpolitischen Nachkriegszäsur ergeben.

\section{Zur politischen Ökonomie des doppelten Deutschland}

Ende der achtziger Jahre sahen sich die entwickelten Geld- und Marktwirtschaften einer Kombination aus zyklischer und struktureller Krisenlagen konfrontiert. Dem - im Herbst 1982 bzw. Frühjahr 1983 begonnenen langanhaltenden, wenn auch auf vergleichsweise niedrigem Niveau anhebendem Aufschwung der kapitalistischen Weltwirtschaft waren die An- 
triebskräfte verloren gegangen. Angeschoben durch eine Renaissance neoliberaler Umverteilungspolitiken in den USA sowie in Japan und Großbritannien und die kreditexpansiven Wirkungen entregulierter nationaler wie vor allem internationaler Geld- und Kreditmärkte wurde die achtziger Jahre hindurch ein fragiler internationaler Wachstumsprozeß initiiert, dessen Kehrseiten - insbesondere die hohen Schuldendienstverpflichtungen aus stark ausgeweiteten Kreditengagements sowie spekulativer Überhitzungen und Volatilitäten der Wechelkurse - sich in der globalen Ökonomie immer stärker bemerkbar machten. Rückläufige Kapazitätsauslastungen, sinkende Zuwachsraten der Produktion, steigende Arbeitslosenzahlen, polarisierte Handels- und Leistungsbilanzen bei ruickläufigen Zuwachsraten des Welthandels und instabile Wechselkurse bestimmten zunehmend das Bild (vgl. dazu genauer Hübner 1988).

Eine Ausnahme stellte allein die Bundesrepublik dar: dank des wirtschaftsund deutschlandpolitisch erzeugten Einheitsbooms, dessen Nachfragesogwirkungen sich hauptsächlich in den alten Bundesländern niederschlugen, konnte sich die westdeutsche Wirtschaft von den in allen OECD-Ökonomien spätestens ab 1990 rückläufigen Zuwachsraten des Bruttosozialprodukts abkoppeln. Nachdem das reale BSP im Jahresdurchschnitt 1982/1989 gerade einmal um 2,1\% anstieg, wurden nach der Herstellung der Wirtschafts- und Währungsunion 1990 bzw. 1991 Zuwachsraten von 4,9 bzw. 3,6\% verzeichnet. Berechnungen zufolge verstärkten die wirtschaftspolitischen Konsequenzen des Herrn Kohl (Hagemann) allein im Jahre Eins nach der Wirtschafts- und Währungsunion das westdeutsche Wachstum um 2,3\% (Hagemann 1993, 26). Demgegenüber blieben seit 1990 die Zuwachsraten der bundesdeutschen Exporte als Reflex der rückläufigen ausländischen Nachfrage nach bundesdeutschen (Investitions-) Gütern und der geld- und kreditpolitisch verursachten Aufwertung der D-Mark sowie des Auftretens neuer kompetitiver Anbieter auf den Weltmärkten hinter den Zuwächsen der gesamtwirtschaftlichen Produktion zurück (vgl. SVR 1992, 77ff.). Zwar konnte das vereinigte Deutschland - um den Preis einer Umkehrung der bundesdeutschen Leistungsbilanz in ein Defizit von 33 Milliarden D-Mark im Jahr 1991 - kurze Zeit als internationale Konjunkturlokomotive fungieren, doch reichten diese Impulse nicht aus, um in der Weltwirtschaft einen sich selbst tragenden Aufschwung zu generieren. Mit nachlassenden Wirkungen des Einheitsbooms verlor die alte Bundesrepublik zunehmend ihre konjunkturpolitische Sonderrolle und ordnete sich seit Anfang 1993 in die Reihe stagnierender OECD-Ökonomien ein:

$\gg$ Die weiterhin hohe, aber nun deutlich langsamer zunehmende Nachfrage aus den neuen Bundesländern reichte nicht mehr aus, die rückläufigen Lieferungen ins Ausland sowie die durch den Realkaufkraftentzug (höhere indirekte Steuern, Preissteigerungen) geschwächte Konsumnachfrage im Inland überzukompensieren. Da sich die in der Boomphase aufgebauten Produk- 
tionskapazitäten nun als zu hoch erweisen und nicht mehr ausgelastet werden können, ist eine weitere Ausdehnung der Investitionen über das ohnehin hohe Niveau hinaus überfluissig « (Prognos 1993, 135).

In den Fünf Neuen Ländern (FNL) erzeugte die mit der Wirtschafts- und Währungsunion vom Juli 1990 erfolgte Überstülpung bundesdeutscher Wirtschafts- und Währungsverhältnisse einen tiefgreifenden Einheitsschock, der sich in eine akzelerierende Anpassungskrise mit drastisch schrumpfenden Kapazitäten, De-Industrialisierungstendenzen und steigenden Arbeitslosenzahlen wandelte. Die Industrieproduktion der FNL fiel bereits einen Monat nach der Wirtschafts- und Währungsunion auf 60\% des Durchschnittniveaus des ersten Halbjahres 1990; bis Ende 1991 betrug sie nur noch ein Drittel des Ausgangsniveaus. Als Folge stieg die effektive Arbeitslosenquote (incl. von Arbeitskräften in ABM- und Umschulungsprogrammen sowie in äquivalente Vollzeitarbeitslose umgerechnete Kurzarbeiter) bis Ende 1991 auf mehr als 30\% an (vgl. Sinn/Sinn 1992, 34) - und dies trotz weiter anhaltender Westmigration von Arbeitskräften und hohen Bestandssubventionen für Treuhandunternehmen.

Mit Blick auf zentrale ökonomische Faktoren ist der Abstand zwischen den alten und den neuen Bundesländern bis heute kaum geringer geworden: Mit einem 20\%-Anteil an der gesamtdeutschen Bevölkerung erwirtschafteten die FNL im Jahr 1992 gerade einmal 7,8\% des gesamtdeutschen BSP (Ganßmann 1993, 194). Überbrückt wurde diese Kluft mit öffentlichen Transfers, die sich in Nettorechnung (von den Bruttotransfers des Jahres 1992 bspw. werden Steuer- und Beitragseinnahmen der FNL subtrahiert) 1992 auf 125 und 1992 auf 150 Milliarden D-Mark addierten (vgl. SVR 1992, 144). Die gesamtwirtschaftliche Absorption der FNL konnte aufgrund dieses Mechanismus bspw. im Jahr 1992 mit 432 Milliarden D-Mark fast doppelt so hoch ausfallen wie das BIP der FNL, wobei $94 \%$ dieser Güterlücke durch Lieferungen aus Westdeutschland gefüllt wurden (vgl. Prognos 1993, 135). Anders ausgedrückt: Die Bevölkerung der FNL lebt weit über ihre Verhältnisse. Niederschlag findet diese Entkoppelung von ökonomischer Leistungsfähigkeit und Verfügung über Ressourcen im Anstieg der Kreditaufnahme von Bund und Gebietskörperschaften. Das jährliche Defizit inklusive aller Nebenhaushalte sowie der Sozialversicherung stieg von 151 Milliarden im Jahr 1991 (5.3\% des BIP) auf 215 Milliarden D-Mark im Jahr 1992 an (7\% des BIP) (vgl. SVR 1992, 141). Der hohe Finanzbedarf der FNL-Transformation und das rasche Tempo der staatlichen Kreditaufnahme wurde von den Kapital- und Finanzmärkten in Gestalt steigender Realzinssätze antizipiert, die von der Deutschen Bundesbank aus stabilitäts- wie allokationspolitischen Gründen noch gefördert wurden. Die Konsequenz war eine rapide Verteuerung kreditfinanzierter Investitio- 
nen - und eine forcierte Umverteilung der Einkommen zugunsten der nationalen wie internationalen Vermögensbesitzer. Verlangsamte Modernisierung der Produktionsanlagen in den alten wie den neuen Bundesländern und Verschärfung der Einkommensungleichverteilung sind die Folgen.

In diesem Rahmen Lohnpolitik zu betreiben, erwies sich als schwieriges Geschäft. Die unter der marktorthodoxen Maxime »one market - one price « von durch westdeutsche Repräsentanten dominierten Unternehmensverbänden wie Gewerkschaften (mit staatlicher Unterstuitzung) eingeschlagene Lohnangleichungspolitik bewirkte zusammen mit der nur mäßig steigenden industriellen Arbeitsproduktivität eine kräftige Steigerung der ostdeutschen Lohnstückkosten - und damit eine Profitquotendeflation der FNL-Betriebe, die ihrerseits die Investitionståtigkeit bremste und neue Transfers für die steigende Zahl von Arbeitslosen nach sich zog. Nach Berechnungen des DIW bewegte sich 1992 die Kostenbelastung der ostdeutschen Industrie aufgrund der gegenüber der Arbeitsproduktivität schneller steigenden Nominallöhne auf einem doppelt so hohen Niveau wie in der westdeutschen Industrie (DIW 1993, 133). Der steile Anstieg der Arbeitskosten verdankt sich dabei keineswegs vorrangig einer überzogenen gewerkschaftlichen Lohnpolitik, sondern ist selbst die Konsequenz des mit der deutschen Einheit verknüpften Entwicklungsgefälles zwischen Ost und West. Häufig werden die überproportionalen Nominallohnsteigerungen mit dem law of one price begründet, das sich in einer vereinheitlichten Wirtschaft sozusagen automatisch Geltung verschaffe. Von einem Automatismus kann dabei jedoch nicht gesprochen werden. Auf einem fiktiven nationalen Arbeitsmarkt kommt es - modelltheoretisch gesprochen - dann zu einem einheitlichen Preis der Ware Arbeitskraft, wenn vollständige sektorale wie regionale Mobilität der Arbeitskraft existiert, und diese von den Lohnabhängigen auch praktiziert wird. In diesem Falle kommt es zu Arbitrageprozessen, wodurch in ehedem Niedriglohnregionen die Arbeitskraft verknappt und in ehedem Hochlohnregionen das Arbeitskraftvolumen ausgeweitet wird. Steigende bzw. rückläufige Zuwachsraten der Nominallöhne wären dann die Folge. Obwohl nun in den FNL eine ausgeprägte Mobilität der Arbeitskräfte i.S. einer Westmigration festzustellen ist, kann doch aufgrund der rasanten Freisetzungsdynamik im Zuge der De-Industrialisierungsprozesse von einer Verknappung der Ware Arbeitskraft nicht gesprochen werden (vgl. zu den einschlägigen Daten Nolte/Ziegler 1994). Die Durchsetzung des law of one price wurde faktisch den Tarifparteien mit dem Instrument des Flächentarifvertrages zugewiesen. Mit Blick auf die große Zahl der konkursgefährdeten Treuhandbetriebe sprechen Akerlof et. al. von einem end game-Charakter der Lohnverhandlungen: 
»In an end-game situation, where an industry is clearly dying, investment will be low whatever the level of wages since firms have more capital than needed. As a result, the elasticity of demand for labor is low and labor has an incentive to raise wages and appropriate the quasi rents of the firm « (Akerlof et.al. 1991, 61).

Auch ist in Rechnung zu stellen, daß die für den Fall von Entlassungen bereitgestellten Lohnersatzleistungen an die Höhe der Nominallöhne gekoppelt sind, so daß es auch unter diesem Aspekt für die Noch-Beschäftigten respektive ihre gewerkschaftliche Interessenvertretungen rationale Gründe für möglichst hohe Lohnabschlüße gegeben hat. Und schließlich ist daran zu erinnern, daß allenthalben von einer hohen Kapitalzufuhr und schnell steigenden Zuwachsraten der Arbeitsproduktivität im Rahmen der avisierten High-Tech-Ökonomie in den FNL ausgegangen wurde. Diese Vorstellung mag auch verantwortlich dafür zeichnen, daß die Unternehmerverbände die Lohnangleichungspolitik über einen langen Zeitraum mitgetragen haben. Die faktischen Wirkungen der forcierten Lohnangleichungspolitk sahen freilich anders als erwartet aus. Während nämlich die Kaufkraft- und Nachfrageeffekte rasch steigender Nominallöhne vor allem in den alten Bundesländem oder in anderen Regionen der Weltwirtschaft anfielen und dort kurzfristig das Wachstums- und Beschäftigungsniveau sowie die Nominallöhne erhöhten, verharrten die Kosteneffekte allein in den FNL. Weil infolge des gebremsten Investitionsverhaltens des privaten Unternehmenssektors und mangelnder bzw. fehlender sozialer wie technischer Infrastrukturen die Zuwachsraten der Arbeitsproduktivität nicht nur mit dem Wachstum der Löhne nicht Schritt halten konnten, sondern gerechnet als Stundenproduktivität - sogar abgesunken sind (vgl. dazu Hagemann 1993a, 100), wurde die internationale Wettbewerbsposition der in den FNL ansässigen Betriebe geschwächt.

Das politische Versprechen, einheitliche Lebensbedingungen im gesamten Deutschland in kürzester Frist per Lohn- und Sozialpolitik herzustellen, war ökonomisch nicht einzulösen. Ohne akkommodierende staatliche Maßnahmen war die Lohnpolitik mit dieser Aufgabe überfordert. Auf welch sandigen Grund diese politische Botschaft gebaut war, zeigt schon eine einfache Kalkulation. Geht man optimistischerweise davon aus, die durchschnittlichen Bruttolöhne der FNL hätten im Jahr $199150 \%$ der in den alten Bundesländern betragen und die westlichen Bruttolöhne würden bis zum - willkürlich gewählten - Stichjahr 1995 um durchschnittlich 4\% ansteigen, dann wären in den FNL jahresdurchschnittliche Steigerungsraten der Bruttolöhne um fast $24 \%$ notwendig, um bis zum Stichjahr eine Angleichung der Bruttolöhne erreicht zu haben (Kalmbach 1993, 125). Wettbewerbspolitisch würde ein solcher Anstieg eine mindestens genau so hohe Steigerungsrate der Arbeitsproduktivität erfordern - eine recht waghalsige Voraussetzung, die angesichts des gemessen an den Wachstumserforder- 
nissen relativ gemäßigten Investitionstempos als nicht erfüllt bezeichnet werden darf. Erfüllt haben sich aber auch nicht die Hoffnungen der Gewerkschaften, sich in den FNL schnell als machtvolle Arbeitnehmerorganisationen zu etablieren und ein allseits befürchtetes Lohn- und Sozialdumping gegenüber den alten Bundesländern zu unterbinden. Die De-Industrialisierungsprozesse und der damit verbundene enorme Arbeitsplatzabbau haben den Gewerkschaften ihre originäre Klientel entzogen und sie in bloß defensive Abwehr- und Erhaltungsaktionen getrieben, die in zunehmenden Maße mit Interessen ihrer Westklientel konfligieren. Staatliche Wirtschaftspolitik und Unternehmen nutzen heute die selbst mitverursachte DeIndustrialisierungskrise in den FNL, um ein flächendeckendes roll back der Arbeitsbeziehungen in Gesamtdeutschland in Angriff zu nehmen.

Die Nettotransfers zugunsten der FNL zeitigen nicht allein haushalts- und gestaltungspolitische Konsequenzen in Gesamtdeutschland. Sie haben auch unmittelbare Auswirkungen auf die allokative Struktur und insbesondere die internationale Wettbewerbsfähigkeit der deutschen Industrie. Orientiert man sich an der prinzipiellen Unterscheidung zwischen international handelbaren und nichthandelbaren Gütern, wobei letztere allgemein solche Güter sind, deren Nachfrage in einem Land durch eigene Produktion bedient werden muß, dann läßt sich zeigen, daß die einkommenswirksamen West-Ost-Transfers nach einer ersten Phase nachholenden Konsums überproportional den Bereich der nichthandelbaren Güter begünstigt und so die sektorale Struktur zuungunsten der handelbaren Güter - hauptsächlich: Industriewaren - beeinflußt haben: "Die westdeutschen Unternehmen haben sich auf den ostdeutschen Markt konzentriert und dabei die Auslandsmärkte vernachlässigt. Was bisher international handelbar war, wurde durch die deutsche Vereinigung .... quasi zu einem nichthandelbaren Gut « (Siebert 1993, 250). Für eine exportorientierte Wirtschaft wie die der Bundesrepublik verkörpert eine sektorale Reallokation zu Lasten handelbarer Güter, die in starker internationaler Konkurrenz stehen, dann ein Problem, wenn die quantitative Reduktion mit einer relativen qualitativen Verschlechterung der Angebotsbedingungen dieses Sektors einhergeht. Indirekter Indikator für eine derartige Verschlechterung ist die Entwicklung der Arbeitsproduktivität, deren Zuwachsraten sich die gesamten achtziger Jahre hindurch auf einem relativ niedrigen Niveau bewegten (vgl. SVR 1992, 311). Entscheidend zu diesem Prozeß sektoraler Reallokation beigetragen haben weniger die staatlichen Transfers als solche, ais vielmehr ihre überwiegend konsumtive Verwendung, die wiederum die Folge gescheiterter Transformationsstrategien von Politik und Wirtschaft darstellt, die mit - konsumtiven - sozialstaatlichen Maßnahmen kompensiert werden (müssen). Aus höheren konsumtiven Transfers in den Osten Deutschlands folgt unter den 
aktuellen Bedingungen finanzpolitischer Restriktionen, daß das Volumen staatlich-investiver Ausgaben in den alten Bundesländer zurückgeht. Da mit Kaldor gilt, daß die Steigerung der Arbeitsproduktivität in hohem MaBe bedingt ist durch das Tempo der Kapitalakkumulation i.S. produktiver Investitionen, und wenn weiter gilt, daß tiefgreifende technische und organisatorische sowie wettbewerbspolitische Umbrüche von staatlichen Unterstützungen (»developmental state«) flankiert werden müssen, dann läßt sich daraus folgern: Je disproportionaler das Verhältnis zwischen investiven und konsumtiven Transfers und je geringer bzw. ineffizienter unternehmerische und staatliche Leistungen zugunsten des Innovationsprozesses ausfallen, desto geringer entwickelt sich das Tempo der Kapitalakkumulation und desto kleiner werden die Zuwachsraten der Arbeitsproduktivität.

In Rechnung zu stellen ist in diesem Zusammenhang allerdings, daß die Produktivitätsschwäche der westdeutschen Industrie keineswegs eine direkte und unmittelbare Folge des Einheitsprozesses ist. Tatsächlich reicht der Produktivitätsrückstand industrieller Schlüsselbranchen wie des Automobilbaus und zunehmend auch des Maschinenbaus auf die frühen achtziger Jahre zurück. Wie vergleichende Produktivitätsstudien zeigen können, verdankt sich die Produktivitätskrise nicht in erster Linie höheren Kostenstrukturen, relativ geringer Automatisierungsniveaus oder etwa ausgeweiteter konsumtiver Transfers des Staates. Verantwortlich zeichnet vielmehr eine tradierte Form von politics in production des Unternehmenssektors. Für den Automobilbau wird beispielsweise

»der Kostenvorteil japanischer Fahrzeuge zu 38\% durch höhere Effizienz in der Fertigung und in der Montage der Fahrzeuge, zu 20\% durch Lohndifferenzen, zu 24\% durch Währungsrelationen und zu 18\% durch Steuern und Gebiihren begründet« (Naschold 1991, 114).

Unzureichende unternehmerische Anpassungsstrategien an gewandelte technologische wie arbeitsorganisatorische Anforderungen und eine Verschlechterung der Innovationsfähigkeit haben dazu beigetragen, daß der westdeutsche Weltmarktanteil für Hochtechnologieprodukte zwischen 1970 und 1990 von 16 auf 13\% abgefallen ist (vgl. Jens 1993). Der durch die konkrete Form des Einheitsprozesses beförderte nachholende Konsum der FNL-Bürger hat mit seinen kapazitätsauslastenden Effekten dazu beigetragen, daß breite Bereiche des westdeutschen Unternehmenssektors an ihren im internationalen Vergleich überholten Management-und Investitionspraktiken festgehalten haben:

»Bereits durch die deutsche Vereinigung ist das Spezialisierungsniveau der deutschen Industrie bei technologieintensiven Gütern insgesamt zurückgegangen, was ausschließlich auf die rückläufige Spezialisierung im Bereich der höherwertigen Technik zurückzuführen ist« (Welsch 1994, 17). 
In einer im Auftrag des BMFT durchgeführten jüngeren Analyse zur technologischen Wettbewerbsfähigkeit der deutschen Indüstrie wurde ermittelt, daß sich verglichen mit dem alten Gebietsstand seit Anfang der neunziger Jahre der technologische Rückstand zu Japan auf vielen Gebieten deutlich vergrößert und sich der altbundesdeutsche Vorsprung zu anderen Anbietern ebenso deutlich verringert habe (nach Welsch 1994).

Setzt sich die Innovations- und die damit verknüpfte Produktivitätsschwäche fort, dann darf mit einer weiteren Verschlechterung der bundesdeutschen Wettbewerbsposition auf den Weltmärkten gerechnet werden. Das entwicklungsoptimistischen Positionen zufolge aus der Deutschen Einheit erwachsen sollende »Deutsche Wirtschaftswunder« (Seppain 1993, 83) dürfte unter solchen Umständen nicht eintreten. Vielmehr könnte eine gegenläufige Entwicklung eintreten: Gerade weil die FNL sich in einem vicious circle verfangen haben, besteht die Möglichkeit negativer Rückkopplungsschleifen für die alten Bundesländer. Geht man nämlich davon aus, daß der wirtschaftliche Erfolg des Modell Deutschland nicht zuletzt in einer strategischen Unterbewertung der D-Mark als Ergebnis einer spezifischen gesellschaftlichen Stabilitätskonstellation gründet, dann unterminieren die zumindest mittelfristig hohen West-Ost-Transfers und damit einhergehende staatlichen Defizite sowie nominale wie reale Aufwertungstendenzen im Zuge hoher Kapitalimporte diese Erfolgssäule (s. Spahn 1993). Sprengkraft entwickelt hat die spezifische ökonomische Form der Herstellung der deutschen Einheit für das Europäische Währungssystem (EWS), und letztlich auch für die weitere Dynamik des Maastricht-Prozesses: Ins Sperrfeuer öffentlicher Kritik geraten ist die Haushaltspolitik der Bundesregierung sowie die Geldpolitik der Deutschen Bundesbank. Der extreme Anstieg der Kreditaufnahme der öffentlichen Haushalte und die offensichtlichen Transformationsprobleme in den FNL wurden von den Geld- und Kreditmärkten schnell in antizipative Zinssteigerungen - insbesondere für kurzfristiges Geldkapital - übersetzt, die von der Bundesbank mit geld- und kreditpolitischen Maßnahmen verstärkt wurden. Zwar lag der langfristige Realzinssatz mit 4\% zu Beginn des Jahres 1992 fast auf dem gleichen Niveau wie im Jahr 1988, doch unterschieden sich die makroökonomischen Konstellationen beider Jahre deutlich: Zeichneten sich 1988 erste Rezessionstendenzen $a b$, so war die Krise - insbesondere in der Internationalen Politischen Ökonomie - im Jahr 1992 bereits in vollem Gange. Zu erwarten gewesen wäre mithin eine Senkung der Realzinsen - und zwar der langwie kurzfristigen Sätze. Der hohe Kapitalbedarf der öffentlichen Haushalte (inklusive der ausufernden Nebenhaushalte) sowie sich verstärkende Inflationstendenzen ließen in den Augen der Bundesbank - sowie des internationalen spekulativen Geldkapitals - in der Bundesrepublik freilich eine sol- 
che Zinsentwicklung nicht zu. Von Anfang bis 1992 bis August 1992 war vielmehr eine stetige Zunahme der Leitzinsen als Reaktion auf die ungebremste Kreditaufnahme des öffentlichen Sektors und einer defizitären bundesdeutschen Leistungsbilanz zu beobachten.

Dank der dominanten Stellung der D-Mark innerhalb des EWS gerieten die Mitgliedsländer in den deutschen Zinssog: Wollten sie einen starken Kapitalabfluß in die Bundesrepublik infolge der dortigen hohen Realzinsen sowie leistungsbilanzbedingter Abwertungserwartungen verhindern, blieb ihnen keine andere Wahl, als eine gleichgerichtete Zinspolitik selbst um den Preis einer Verstärkung der internen Krisentendenzen zu verfolgen. Versuche etwa Frankreichs, Geldmarktzinsen unterhalb des deutschen Niveaus durchzusetzen, blieben erfolglos, und mussten mit gewaltigen Devisenmarktinterventionen der betroffenen Zentralbanken - in diesem Fall sah sich die Banque de France gezwungen, ihre gesamten Devisenreserven einzusetzen, die Bundesbank intervenierte mit 60 Milliarden D-Mark - bezahlt werden (SVR 1993, 138).

Diese geldpolitische Konstellation wiederum bot dem spekulativen Geldkapital der internationalen Märkte die Möglichkeit, das EWS einem höchst ertragreichen »Belastungstest « $z \mathbf{u}$ unterziehen, der schließlich beginnend im August 1992 zu einer faktischen Auflösung des europäischen Fixkurssystems führte. Die drastische Ausweitung der Wechselkursschwankungsbandbreiten von $+1-2,25 \%$ bzw. im Falle Portugals und Spaniens $6 \%$ auf $+/-15 \%$ und das temporäre Ausscheiden Italiens und Großbritanniens aus dem Verbund dürften zwar nicht das Ende der Maastricht-Vereinbarungen darstellen, doch wird der ursprünglich vorgesehene Zeitplan angesichts der faktischen monetären Divergenzen und der Aufweichung des EWS-Fixkurssystems in ein Quasi-Flexsystem realistisch betrachtet nicht eingehalten werden können (so jüngst auch Heine/ Herr 1994). Noch entscheidender dürfte sein, daß diese geldpolitische Episode der Deutschen Einheit die Maastricht-Skeptiker in vielen Ländern der Europäischen Union gestärkt hat, wurde doch mit dem konkreten wirtschaftspolitischen Vollzug der deutschen Einheit deutlich, daß die Bundesrepublik im Zweifelsfalle nationale Interessenlagen höher veranschlagt als gemeinsame internationale ökonomische Interessen.

Anzumerken ist freilich auch, daß die Deutsche Bundesbank durch die ökonomischen Folgen der Deutschen Einheit in eine strategische Sackgasse geraten ist: "Zinssenkungen lassen eine nominale DM-Abwertungstendenz mit der Gefahr verstärkter Preissteigerungen erwarten, Zinserhöhungen eine weitere nominale Aufwertung mit einem auslastungsbedingten Rückgang der Inflationsrate (Spahn 1993, 44). Die Strategie einer Unterbewertung der D-Mark jedenfalls dürfte angesichts der manifesten internen 
Strukturprobleme der neuen Bundesrepublik für längere Zeit nicht mehr gefahren werden können. Damit dürften dann aber auch die seigniorageGewinne entfallen, die in der Vergangenheit aus der hegemonialen Stellung der bundesdeutschen Währung entsprungen sind.

Mauer und Stacheldraht zwischen den ehedem zwei deutschen Staaten sind verschwunden - neu entstanden sind ökonomische Schlagbäume, die - zugespitzt formuliert - nur hochgehen, wenn Waren und Transfers von West nach Ost transportiert bzw. überwiesen werden und mobile, junge Arbeitskräfte sich von Ost nach West bewegen. Das in neuer Form geteilte Deutschland stellt die Politik vor schwierige Probleme: Die Unternehmen der alten Bundesländer stehen in harter Konkurrenz mit hochproduktiven und hochinnovativen Wettbewerbern der Weltwirtschaft. Die in den FNL, ansässigen Betriebe rangieren im internationalen Produktivitätswettbewerb im unteren Mittel und müssen mit ihren vergleichsweise hohen Lohnstïckkosten vor allem mit den neuen Niedriglohnökonomien ost- und mitteleuropäischer Anbieter konkurrieren.

Fasst man der Einfachheit halber die neuen und die alten Bundesländer einmal als »Regionen « auf, dann läßt sich formulieren, daß beide Regionen sich innerhalb völlig unterschiedlicher Segmente der globalen Ökonomie bewegen. Da beide Regionen aber Teile eines nationalstaatlichen Ganzen sind und vor allem mit einer einheitlichen Währung in die globale Ökonomie eintreten, stellt sich für die staatliche Politik - für die Geldpolitik der Zentralbank wie für die Wirtschaftspolitik der Bundesregierung - das Problem, höchst unterschiedliche Anforderungen und Zielkomplexe beider Regionen vereinheitlichen oder zumindest kompatibel machen zu müssen. Die Erfahrungen zeigen, daß eine solche Vereinheitlichung nur ausnahmsweise möglich ist. Stellt man darüberhinaus die (noch existente) Leitwährungsrolle der D-Mark im EWS in Rechnung, werden die Anforderungen an die bundesdeutsche Geld- und Fiskalpolitik noch komplexer: Internationale Handlungserwartungen und widerspruichliche interne Zielkomplexe wären kompatibel zu gestalten.

\section{3. Ökonomische Paradoxien der Einheitspolitik}

Regionale Ungleichheiten des bislang geschilderten Typs können - wie auch empirische Beispiele zeigen - langfristig aufrechterhalten bleiben. Wie schon Myrdal (1957) mit seinem Prinzip der zirkulären und kumulativen Verursachung gezeigt hat, ist es sehr wohl möglich, daß sich eine Region in einem virtious circle und die andere Region zur gleichen Zeit in einem vicious circle bewegen. Fallen in der einen Region firmenexterne und regioneninterne steigende Skalenerträge an und bestehen zwischen beiden 
Regionen ausgeprägte Differenzen im Niveau der Arbeitsproduktivität zugunsten der Region mit steigenden Sk̉alenerträgen, dann weist letztere - bei annähernd gleichen Lohnkosten - einen Vorteil in den Lohnstuickkosten auf, der sich in einen Absatz-, Umsatz- und Profitvorteil übersetzt. Die Region mit steigenden Skalenerträgen entwickelt sich zunehmend auf Kosten der benachteiligten Region und kann ihren Wettbewerbsvorteil ausbauen. Eine gegenläufige Vorstellung ökonomischer Entwicklungsverläufe formuliert die Gerschenkron-Hypothese, derzufolge sich die relative Rückständigkeit eines ökonomischen Raumes dann als Vorteil erweisen kann, wenn dadurch in dem rückständigen Land - möglichst lokal angepasste - institutionelle Innovationen induziert werden, staatliche Interventionen zur sektoralen Kanalisierung von Kapital in die aufzubauenden jungen Industrien erfolgen und die Kapitalgüterindustrie forciert wird (vgl. Fishlow 1987, 519). Eine aktive staatliche Industriepolitik zur Mobilisierung der Chancen relativer Rückständigkeit wurde freilich erst gar nicht in Erwägung gezogen.

Kurz $(1993,78)$ zufolge treffen die Bedingungen des Myrdalschen Prinzip der kumulativen und zirkulären Verursachung recht exakt den deutschdeutschen Fall:

»Aus offensichtlichen historischen Gründen ist Ostdeutschland in einen inferioren Pfad wirtschaftlicher Entwicklung 'eingesperrt'. Unter der Annahme, daß die Bereitschaft der westdeutschen Steuerzahler begrenzt ist, die strukturellen Defizite der ostdeutschen Wirtschaft wettzumachen, scheint für eine Variante des Circulus vitiosus-Szenarios als Vorschau auf die zukünftige ökonomische Entwicklung der neuen Länder die größte Wahrscheinlichkeit zu sprechen«.

Einer dieser offensichtlichen historischen Gründe ist die in der vormaligen DDR vorgenommene Deprivation der Region als verdichteter ökonomischer Raum. Die insbesondere seit den späten sechziger Jahren eingeleitete Politik einer möglichst weitgehenden Internalisierung ökonomischer Transaktionen in die Kombinate hatte zur Folge, daß die bis dahin existenten intraregionalen forward and backward linkages, basierend auf der Existenz traditioneller kleiner handwerklich orientierter Industrieproduktionen, kurzerhand abgeschnitten wurden (vgl. Grabher 1993, 5). Für westliche Unternehmen sind ökonomische Räume dieses Typs nicht in erster Linie Märkte sondern im weitesten Sinne exportorientierte Produktionsstandorte, die sich durch eine Kombination relativ billiger unqualifizierter Arbeit und moderner Massenproduktion insulär erschließen lassen, indem die zentralen Vorleistungen in weit überwiegendem Maße aus den westlichen Ländern antransportiert und vor Ort nur noch zusammengebaut werden. Nicht zuletzt vor dem Hintergrund auch im internationalen Vergleich hoher Kapitalsubventionen drängt sich die Vermutung auf, daß die konstituierten Zweigbetriebe den Charakter von »snatcher « haben (vgl. Berry/Conkling/ Ray 1993, 273ff.), d.h. auf die rasche Nutzung der historisch besonderen Pro- 
duktionsbedingungen von Profit zielen und dann schnell wieder geschlossen werden, wenn sich die komparativ günstigen Produktionsbedingungen beispielsweise infolge rasch steigender Lohnstückkosten und/oder sinkender staatlicher Subventionen verschlechtern.

Derartige Investitionsstrategien können in all den Branchen eine Rolle spielen, die relativ geringe Ausstiegskosten (»exit costs«) aufweisen. Solche Ausstiegsvorteile wiederum können von Unternehmen in einem Milieu verschärfter internationaler Standortkonkurrenz vor allem dann genutzt werden, wenn es bei diesen Investitionen nicht vorrangig um die ErschlieBung von Marktzugängen oder der Sicherung von Markt- und Absatzpositionen geht. In all diesen Fällen läßt sich festhalten, daß sich für die Regionen daraus keinerlei dynamische Effekte ergeben. Tatsächlich ist es genau die Kombination »ererbter «, international nicht-kompetitiver Produktionsbedingungen und fehlgeleiteter wirtschafts- und tarifpolitischer Entscheidungen der Nachwendezeit, die das Argument des vicious circle für die FNL - abseits der durchaus realistischen Möglichkeit einer kleinen Zahl von »Produktivitätsinseln« - so überzeugend machen.

Dieses Argument läßt sich noch mit den zu erwartenden Wirkungen eines stetigen Exports von Arbeitskräften von der peripheren zur zentralen Region bekräftigen. In einer Modellökonomie, in der Arbeit der einzige Produktionsfaktor ist und entweder in der Produktion von Exportgütern oder "Inlandsgütern " (einer Region) eingesetzt wird und alle Einkommen vollständig ausgegeben werden, hängt das produzierte Volumen von Inlandsgütern bei gegebenem Lohnsatz von der Größe des Binnenmarktes ab. Wenn gilt, daß die Größe des Binnenmarktes vom Umfang des Arbeitsangebotes abhängt und weiter gilt, daß die Größe des Binnenmarktes letztlich die Höhe des Lohnsatzes bestimmt, dann bedeutet eine signifikante Arbeitskräftemigration, daß der Binnenmarkt schrumpft und der Lohnsatz sinkt. Geht man im einfachsten Fall weiter davon aus, daß rückläufige Lohnsätze den Anreiz zur Migration erhöhen, dann läßt sich argumentieren, daß Migrationsprozesse selbstverstärkende Wirkungen zeitigen und einen vicious circle entweder generieren oder einen bereits eingeleiteten kumulativen Abwärtsprozeß perpetuieren können (s. dazu Layard/Blanchard/Dornbusch/Krugman 1992, 77ff.).

Angesichts der Abwanderungen aus den FNL, die selbst im Jahr 1992 sich noch in der monatlichen Größenordnung von 10-15000 Personen bewegten (vgl. SVR 1992, 114), erweist sich eine solche Modellargumentation durchaus als realitätsnah. Die FNL erleben seit Öffnung der Mauer einen anhaltenden brain drain, der - wie sich aus Jánossys Analyse (1968) der kapitalistischen »Wirtschaftswunderökonomien« nach dem Zweiten Weltkrieg ableiten läßt - zu einem qualifikatorischen Engpaß der Kapital- 
akkumulation und damit des Wachstums der Arbeitsproduktivität werden kann. Neuere Studien kommen - selbst unter äußerst optimistischen Annahmen hoher Wachstumsraten der FNL - zu der Prognose einer bis etwa 1995 anhaltenden Nettowanderung von Ost nach West in Höhe von etwa 100.000 Personen; bis zum Jahr 2000 soll sich der Nettosaldo auf etwa 50.000 Personen reduzieren, wobei die Mobilitätsbereitschaft bei jüngeren Personen sehr viel höher ausgeprägt ist (Prognos 1993, 35). Periphere ökonomische Räume wie etwa Mecklenburg-Vorpommern werden davon in besonderer Weise berührt sein, da für sie ein Rückgang der Wohnbevölkerung, aufgrund hoher Wanderungszahlen der Bevölkerung bis zum 40. Lebensjahr um fast 400.000, auf nur noch rund 1,5 Millionen Personen im Jahr 2010 geschätzt wird (Prognos 1993, 200).

Es zählt zu den Paradoxien des deutsch-deutschen Einheitsprozesses, daß diese - wenig wünschenswerte - Entwicklungsvariante durch wirtschaftspolitische Maßnahmen befördert wurde, die eigentlich auf die Herausbildung eines völligen anderen Entwicklungstyps zielten. Absicht der konservativliberalen Bundesregierung war es, aus den FNL eine Hoch-Lohn-HighTech-Region zu machen, die in kürzester Zeit den ökonomisch-technischen Modernisierungsgrad der alten Bundesrepublik überholt und gleichsam im Rückkopplungsprozeß dort Erneuerungen der verkrusteten Strukturen bewirken sollte. Das Mittel dazu sollten die Verträge zur Wirtschafts- und Währungsunion und zur Deutschen Einheit sein. Der Kern der Strategie bestand in der sofortigen Einführung einer Struktur der relativen Preise entsprechend dem gewünschten »Endzustand «, wobei die Vorstellung vorherrschte, durch eine solche Schocktherapie die Ausbildung der angezielten Produktionsstruktur und deren Anpassung an die bereits etablierte Preisstruktur zu beschleunigen (vgl. Sinn/Sinn 1993, 193ff.). Massive staatliche Investitionsförderung zur Induzierung eines Kapitalimports in die FNL und eine forcierte Privatisierungspolitik im Rahmen der Treuhandanstalt, die jegliche Sanierungsmaßnahmen zur Anpassung der OstBetriebe an die gewandelte Umwelt explizit ablehnte, verkörperten die Kerninstrumente der theoretisch insbesondere vom Sachverständigenrat empfohlenen und von der konservativ-liberalen Regierung durchgesetzten Strategie.

Der primär nach macht-und wahlpolitischen Überlegungen gewählte Umtauschkurs von der Mark der DDR gegenüber der D-Mark, die nach dem Motto $\gg$ Gleicher Lohn für gleiche Arbeit! (so die Wahlkampfparole von CDU/CSU) forcierte Tarifpolitik sowie die umfassende Liberalisierung der Preise bewirkten in der Tat eine schnelle Veränderung der relativen Preisstruktur zuungunsten vor allem all der Sektoren und Betriebe, die nicht in der Lage waren, mit ihrem zu Weltmarktpreisen bewertetem Absatz wenig- 
stens die variablen Kosten zu decken. Faktisch bewirkt wurde damit ein Strukturwandel, der darauf hinauslief, daß - wie durchaus gewünscht viele Altindustrien entweder ganz aus dem Produktionsspektrum der FNL verschwunden sind oder auf kleine Bereiche reduziert wurden. Es handelt sich dabei jedoch um einen passiven Strukturwandel: Den durch die geltenden relativen Preise induzierten Entwertungen des Kapitalstocks stehen nur höchst unzureichende Neuzugänge zum Kapitalstock gegenüber. Im Jahr 1992 konnten zwar die privaten Investitionen des Unternehmenssektors auf 60 Millarden D-Mark gesteigert werden. Im Pro-Kopf-Vergleich mit der alten Bundesrepublik liegt die Investitionsrate jedoch erst bei 65\% (SVR 1992, 93). Um angesichts des hohen Entwertungstempos des Kapitalstocks die zur Steigerung der Arbeitsproduktivität und Wettbewerbsfähigkeit notwendigen Zuwachsraten des Nettokapitalstocks zu erreichen, müssten die jährlichen Neuinvestitionen freilich sehr viel höher ausfallen als in den alten Bundesländern. Eine wesentliche Bedingung jeder Hoch-Lohn-HighTech-Strategie, nämlich eine hohe und stetige Rate der Kapitalakkumulation, ist damit nicht erfüllt.

Der Verzicht auf eine Politik des aktiven Strukturwandels, die klare und eindeutige Kriterien zu entwickeln hätte, welche Sektoren wachsen und welche schrumpfen oder ganz verschwinden sollen, hatte eine ganze Reihe paradoxer Folgewirkungen. Die Einführung weltmarktorientierter Kostenund Preisstrukturen bei gleichzeitigen sozial- wie tarifpolitisch vorangetriebenen Angleichungstendenzen zwischen alten und neuen Bundesländern produzierten zwar - wie gewünscht - Produktionsstillegungen, sie erzeugten aber zugleich hohe Freisetzungen von Arbeitskräften, denen keine Kompensationseffekte gegenüberstanden. Um den mit den hohen Arbeitslosenzahlen gefährdeten sozialen Frieden zu gewährleisten, wurden die Betriebe - vor allem aus Mitteln der Treuhandanstalt - mit einem wahren Regen von Subventionen überhäuft. Die auf strukturbeschleunigenden Wandel zielenden Maßnahmen der Hoch-Lohn-High-Tech-Strategie produzierten mithin simultan die Notwendigkeit der Einführung strukturerhaltender Maßnahmen. Diese Selbstblockade staatlichen Handelns wurde durch den Investitionsattentismus des westlichen Unternehmenssektors noch verstärkt. Damit die FNL bis zum Jahr 2000 80\% der Durchschnittsproduktivität der alten Bundesländer erreichen, wurden von einschlägigen Modellrechnungen für einen Zehnjahreszeitraum private Nettoinvestitionsausgaben zwischen 1,5 Billionen und 800 Milliarden D-Mark veranschlagt (Barth 1993, 185ff.).

Von entsprechenden jährlichen Investitionen zwischen 150 und 80 Milliarden D-Mark sind die tatsächlichen privaten Investitionsausgaben, wie bereits gezeigt, weit entfernt. Dafür verantwortlich zeichnen nicht allein 
Mängel des deutschen Einheitsvertrages mit seinen investitionshemmenden und bürokratischen Regelungen. Entscheidend sind vieimehr die privaten Verwertungskalküle, die die FNL als Produktionsstandort mit anderen Produktionsstandorten in einen Vergleich setzen. Dabei spielen die immer wieder angeführten (zu) hohen Tariflöhne (so insbesondere Sinn/Sinn, 1993) in den FNL nur eine Rolle neben anderen. Aus effizienz- wie humankapitaltheoretischer Sicht läßt sich sogar argumentieren, daß die hohen Lohnabschlüsse dringlich erforderlich waren, um Humankapitalinvestitionen zu schützen und die Motivation zu fördern (so etwa Schwedler 1993, 109). Ein solches Argument gewinnt jedenfalls dann an Gewicht, wenn die wirtschaftspolitische Strategie nicht darauf zielt, mit den Niedriglohnökonomien der Großregion, den ost- und mitteleuropäischen Transformationsökonomien, in den Wettbewerb treten zu wollen. Eine solche Zielsetzung wäre höchst fatal, wie schon ein schneller Blick auf die Realität komparativer Lohnniveaus zu zeigen vermag. Betrug in den neuen Bundesländern im Jahr 1990 der durchschnittliche gesamtwirtschaftliche Stundenlohn etwa 6 US-Dollar, so beliefen sich die entsprechenden Stundenlöhne in Polen auf 0.7, in Ungarn auf 0.8, in Bulgarien auf 0.2, in Rumänien auf 0.6 und im europäischen Teil der GUS auf 0.9 US-Dollar (Layard/Blanchard/Dornbusch/Krugman 1992, 2). Zwar dürfte in den FNL das durchschnittliche Niveau der Arbeitsproduktivität höher als in diesen Niedriglohnökonomien ausfallen, doch deuten die in den letzten Monaten in Gang gekommenen Produktionsverlagerungen deutscher wie internationaler Unternehmen in ost- und mitteleuropäische Transformationsökonomien darauf hin, daß in die Niedriglohnökonomien technische »Produktivitätsinseln« exportiert werden, die in Kombination mit niedrigen-Löhnen Lohnstückkosten erzeugen, die die vergleichbarer Produktionen in den FNL weit unterschreiten. Auch wenn der auf die ost- und mitteleuropäischen Transformationsökonomien entfallende Anteil deutscher Direktinvestitionen mit mittlerweile 5,5\% noch äußerst gering ist, verweist die seit $1990 \mathrm{zu}$ registrierende Zunahme von 251 Mio. auf 1,5 Mrd. DM (vgl. SVR 1993, 53) doch, daß die deutsche Industrie die Produktionsverlagerungschancen allmählich zu nutzen weiß. Angesichts solcher Entwicklungen würde jeder Niedriglohnwettbewerb die FNL in eine industrie- wie entwicklungspolitische Sackgasse führen. Verstärkt und verfestigt würde so genau der sektorale und regionale Dualismus zwischen alten und neuen Bundesländern, den zu überwinden alle politischen Akteure angetreten sind.

Die markttheoretisch begründete und legitimations- wie gesellschaftspolitisch gewünschte Lohnangleichungsstrategie hätte nur dann keine negativen Investitions-trade offs gezeitigt, wenn über entsprechende investive und arbeitsorganisatorische Maßnahmen das durchschnittliche Tempo der 
Arbeitsproduktivität noch rascher als die tariflichen Löhne gestiegen wäre. Dies wiederum hätte einen beschleunigten Ausbau und eine gezielte Modernisierung der technischen und sozialen Infrastruktur in den FNL zur Voraussetzung gehabt, also gerade eine gezielte Produktion solcher öffentlicher Güter, auf die Marktgesellschaften in hohem Maße angewiesen sind. Auch wäre es notwendig gewesen, die vordem breit ausgebauten Forschungs- und Entwicklungsaktivitäten für den Umbau zu nutzen. Faktisch wurde eine genau gegenläufige Politik verfolgt: Seit der Herstellung der deutschen Einheit wurde der FuE-Unterbau auf weniger als $20 \%$ des ehemaligen Bestandes geschrumpft (Prognos 1993, 151). Im globalen Standortwettbewerb erweist sich dieser marktkonservative Politikansatz, der die staatliche Rolle als Entwicklungsagentur verneint, immer mehr als Wettbewerbshindernis. Wie empirische Untersuchungen zeigen, verlieren im Zuge der Globalisierung ökonomischer Handlungen nationalstaatliche Wirtschaftspolitiken keineswegs an Bedeutung. Im Gegenteil: Der komparative Vorteil nationalstaatlich organisierter ökonomischer Räume ist in hohem Maße das Ergebnis differenzierter staatlicher Politiken und gesellschaftlicher Regulationsformen (s. dazu etwa Porter 1990).

\section{Kognitive Dissonanzen}

Kognitiver Bezugspunkt der von der konservativ-liberalen Regierung in Gang gesetzten DDR-Transformation war das »Wirtschaftswunder « der alten Bundesrepublik. Völlig verdrängt wurde seitens der politischen Akteure, daß die Bedingungen des westdeutschen Restaurationsweges nach dem Zweiten Weltkrieg sich völlig von den Bedingungen unterscheiden, die heute in den Fünf Neuen Ländern anzutreffen sind. Dies betrifft nicht nur die im ersten Falle trotz Kriegszerstörungen im Vergleich sehr viel höhere Qualität des Kapitalstocks hinsichtlich der technischen Standards und des Alteraufbaus (vgl. dazu Schmieding 1990), die in kürzester Frist hohe marginale Kapitalproduktivitäten erlaubte. Entscheidende Unterschiede liegen vor allem in den Strukturkonstellationen der Weltwirtschaft sowie im $\gg$ Rich uncle «-Faktor (Economist), dem die FNL sich ausgesetzt sehen. Während die kapitalistische Restauration der Bundesrepublik parallel begleitet wurde von der Rekonstruktion der kapitalistischen Weltwirtschaft als eines Systems des embedded liberalism, wobei insbesondere die bundesdeutsche Investitionsgüterindustrie von der Öffnung des Welthandels profitieren konnte, erfolgt die Transformation der ehemaligen DDR unter Bedingungen einer bereits voll entfalteten Weltwirtschaft, in der zentrale Marktsegmente hochkompetitiv besetzt sind. Die strategische Absicht, in den Fünf Neuen Ländern sozusagen das Industrialisierungsmodell der alten 
Bundesrepublik in verkleinerter Form zu imitieren und die dort ansässigen bzw. anzusiedelnden Unternehmen über Marktincentives für die gleichen internationalen Marktsegmente zu motivieren und fördern, musste auf ökonomische wie politische Widerstände stoßen. Insbesondere in der Konstellation einer tiefgreifenden weltwirtschaftlichen Krise bemühen sich marktetablierte Unternehmen, ihre Marktpositionen gegenüber aktuellen wie neuen Wettbewerbern abzusichern. Sichtbare wie unsichtbare Marktzutrittsbarrieren werden aufzubauen versucht, durch flexible Reaktion auf neue Marktgegebenheiten werden Produktionsnischen geschlossen und schließlich werden alle Produktivitätsreserven bemüht, um kostenseitig die Nachfrage- und Umsatzrückgänge zu kompensieren. Für Newcomer wie die FNL sind dies höchst ungünstige Marktzutrittsbedingungen, insbesondere dann, wenn die politischen Akteure zur Bewältigung dieser Aufgabe in erster Linie auf den Markt setzen und auf eine aktive staatliche Entwicklungsstrategie weitgehend verzichten.

Diese Konstellationen der Weltwirtschaft gelten für alle ost- und mitteleuropäischen Transformationsökonomien in gleichem Maße. Die Besonderheit der ehemaligen DDR besteht in dem »Rich uncle «-Faktor: Die direkt anfallenden finanziellen Transformationskosten werden hauptsächlich von den westlichen Bundesländern übernommen, wobei die Kostenübernahme eingetauscht wird gegen die politische Bestimmung der Transformationsgestaltung. Der dabei gewählte Weg eines Institutionentransfers von der alten Bundesrepublik in die FNL erweist sich heute allerdings teilweise als Entwicklungsbarriere, wird damit doch eine Regulationsweise in eine Regionalökonomie implantiert, die dem dort existenten Akkumulationsregime nicht angepasst ist. Diese besondere Form der Enteignung von Souveränität kennen die anderen Transformationsökonomien nicht. Ökonomisch hat sich dieser ungleiche politische Tausch bislang in einem vicious circle der neuen Bundesländer niedergeschlagen. Politisch ist er die Basis für die kognitiven Dissonanzen, die sich mittlerweile in breiten Bevölkerungsschichten bemerkbar machen. Die Sehnsüchte nach Freiheit, wie sie sich in den Ereignissen des Jahres 1989 ausgedrückt haben, erfahren Enttäuschungen. Auch dies könnte ein Grund sein, die Umbaustrategie für die Fünf Neuen Länder noch einmal zu überdenken.

\section{Literatur}

Barth, A. (1993): Produktivitätsentwicklung und Investitionsbedarf in Ostdeutschland - Eine Modellrechnung, in: H. Hagemann (Hrsg.): Produktivitätswachstum, Verteilungskonflikte, Beschäftigungsniveau, Marburg.

Berry, B.J.L./Conkling,E.C./Ray,D.M. (1993): The Global Economy. Resource use, Locational choice, and international trade, Englewood Cliffs. 
Cardoso, F.H. (1993): North-South Relations in the Present Context: A New Dependency?, in: Carnoy,M./Castell,M./Cohen,St.S./Cardoso,F.H. (eds.): The New Global Economy in the Information Age. Reflections on our Changing World, Houndmills.

DIW (1993): Deutsches Institut für Wirtschaftsforschung und Institut für Weltwirtschaft, Gesamtwirtschaftliche und unternehmerische Anpassungsprozesse in OstdeutschIand, in: Kieler Diskussionsbeiträge 205/206.

Fishlow, A. (1987): Gerschenkron, Alexander, in: Eatwell, J. et.al. (eds.): The New Palgrave, London.

Ganßmann, H. (1993): Einigung als Angleichung? , in: PROKLA 91, Münster.

Grabher, G. (1993): Instant Capitalism. Starting the Eastern Economy from Scratch?, Paper for the Conference on the Social Embeddedness of the Economic Transformation in Central and Eastern Europe, Social Science Research Center Berlin, September 24-25.

Hagemann, H. (1993): Makroökonomische Konsequenzen der deutschen Einigung, in ders. (Hrsg.): Produktivitätswachstum, Verteilungskonflikte und Beschäftigungsniveau, Marburg.

Hagemann, H. (1993a): On Some Macroeconomic Consequences of German Unification, in: Kurz. H.D. (ed.): United Germany and the New Europe, Aldershot.

Heine, M./Herr, H. (1994): Binnen- und außenwirtschaftliche Koordinationsprobleme nach der deutschen Vereinigung, in: WSI Mitteilungen, Heft 1.

Hübner, K. (1988): Flexibilisierung und die Verselbständigung des monetären Weltmarkts. Hindernisse für einen neuen langen Aufschwung?, in: PROKLA 71, Berlin.

Jánossy, F. (1968): Das Ende der Wirtschaftswunder. Erscheinung und Wesen der wirtschaftlichen Entwicklung, Frankfurt a.M.

Jens, U. (1993): Die fundamentale Innovationsschwäche kann zum Abstieg Deutschlands führen, in; Handelsblatt Nr. 224.

Kalmbach. P. (1993): On Alternative Strategies of Wage Policy in East Germany, in: Kurz, H.D. (ed.): United Germany and the New Europe, Aldershot.

Kurz, H.D. (1993): Deutschland? aber wo liegt es? - Anmerkungen zur mittel- bis längerfristigen Entwicklung des neuen Deutschland, in: H. Hagemann (Hrsg.): 1993.

Layard,R./Blanchard,O./Dornbusch,R./Krugman,P. (1992): East-West Migration. The Alternatives, Cambridge.

Myrdal, G. (1957): Economic Theory and Underdeveloped Regions, London.

Naschold, F. (1991): Internationale Konkurrenz, sektorale Produktionsregime und nationalstaatliche Arbeitspolitik, in: B.Blanke/H.Wollmann (Hrsg.): Die alte Bundesrepublik. Kontinuität und Wandel, Opladen.

Nolte, D./Ziegler,A. (1994): Regionen in der Krise. Regionale Aspekte des Strukturwandels in den neuen Bundesländern, in: WSI Mittteilungen, Heft 1.

Porter, M. (1990): The Comparative Advantage of Nations, New York.

Prognos (1993): Die Bundesrepublik Deutschland 2000 - 2005 - 2010, Basel.

Schmieding. H. (1990): Der Übergang zur Marktwirtschaft: Gemeinsamkeiten und Unterschiede zwischen Westdeutschland 1948 und Mittel- und Osteuropa heute, in: Die Weltwirtschaft, Nummer 2, Kiel.

Schwedler, Th. (1993): Massenarbeitslosigkeit in West- und Ostdeutschland- Folge von RentSeeking-Verhalten und überhöhten Lohnabschlüssen?, in: H. Hagemann (Hrsg.) 1993.

Seppain, H. (1993): European Integration, German Unification and the Economics of Ostpolitik, in: Kurz, H.D. (ed.): United Germany and the New Europe, Aldershot

Siebert, H. (1993): Zu starke Binnenorientierung - Ursache der deutschen Wettbewerbsschwäche, in: Die Weltwirtschaft, Heft 3, Tübingen

Sinn,G./H.-W. Sinn (1992): Kaltstart. Volkswirtschaftliche Aspekte der deutschen Vereinigung, Tübingen

Spahn, H.-P. (1993): Das Dilemma der Wirtschaftspolitik in der Bundesrepublik Deutschland, in: Konjunkturpolitik, Heft $1 / 2$

SVR (Sachverständigenrat) (1992): Jahresgutachten 1992/93, Stuttgart

SVR (Sachverständigenrat) (1993): Jahresgutachten 1993/94, Drucksache 12/6170

Welsch, J. (1994): Innovationsstandort Deutschland - Verpaßte Chancen?, in: WSI Mitteilungen, Heft 1 